\title{
Generic slow relaxation in a stochastic sandpile
}

\author{
Ronald Dickman* \\ Departamento de Física, ICEx, Universidade Federal de Minas Gerais, Caixa \\ Postal 702, 30161-970 Belo Horizonte, Minas Gerais, Brazil
}

(November 20, 2018)

\begin{abstract}
Simulations of a stochastic fixed-energy sandpile in one and two dimensions reveal slow relaxation of the order parameter, even far from the critical point. The decay of the activity is best described by a stretched-exponential form. The persistence probability (for a site not to have toppled up to time $t$ ), also exhibits stretchedexponential relaxation. The results suggest a connection between sandpile models and structural glasses.
\end{abstract}

PACS: 05.40.-a, 05.65.+b, 64.70.Pf, 05.50.+q

Email address: dickman@fisica.ufmg.br 
Sandpile models have attracted much interest in recent years, as paradigms of scale-invariance in the apparent absence of tuning parameters [1, 2, 3, 4], and as intriguing examples of absorbing-state phase transitions [5, 6, 7, 8, 9, 10, 11]. Most studies of sandpiles have focused on the scale-invariant stationary state under slow driving [3], 12], or on scaling properties in the vicinity of the absorbing-state transition [13, 16, 17, 18, 19]. Relatively little attention has been given to the dynamic properties of sandpiles away from the critical point.

A central feature of sandpile models is the presence of a conserved field, the density of particles. This field couples to the activity density, which is the order parameter. When, as in the case of FES, the conserved field is frozen in the absence of activity, the critical behavior is expected to fall in a universality class distinct from that of directed percolation (DP) [13. (In the absence of such a conservation law, DP is generic for absorbing-state phase transitions 114,15. Relaxation of the order parameter to its quasi-stationary value in a sandpile at the critical density is best characterized as a power-law [9], but with certain anomalies in the one-dimensional case [10]. Besides having an effect on the critical behavior, it is reasonable to expect the conservation law to modify relaxation properties away from the critical point as well.

In simple models exhibiting an absorbing-state phase transition, such as the contact process [20], relaxation to the stationary state is expected to be monotonic and exponential, away from the critical point [21. The simulation results reported below show that in sandpiles, off-critical relaxation is considerably slower, following a stretched-exponential or, in certain cases, algebriac decay. Stretched-exponential functions have been reported for avalanche size distributions in experiments on sand and rice piles [22,23], and in granular avalanche models [24], but not, to my knowledge, in the context of sandpile relaxation dynamics.

I study a variant of Manna's sandpile [25], defined on a lattice of $L^{d}$ sites (in $d$ dimensions), with periodic boundaries. The configuration is specified by the number of particles $z_{i}=0,1,2, \ldots$ at each site; sites with $z_{i} \geq 2$ are said to be active. A Markovian dynamics is defined by the toppling rate, which is unity for all active sites, and zero for sites with $z_{i}<2$. When a site $i$ topples, it sends two particles to adjacent sites $\left(z_{i} \rightarrow z_{i}-2\right)$; the particles move independently to randomly chosen nearest neighbors $j$ and $j^{\prime}\left(j, j^{\prime} \in\{i+1, i-1\}\right.$ in the onedimensional case). Thus $j=j^{\prime}$ with probability $1 / 2 d$. The dynamics conserves the number of particles, $N$, which is fixed by the initial configuration.

The system evolves via a sequential dynamics: the next site to topple is chosen at random from a list of active sites; the time increment associated with a toppling is $\Delta t=1 / N_{A}$, where $N_{A}$ is the number of active sites just prior to the event. Initial configurations are generated by distributing $\zeta L^{d}$ particles randomly over the lattice, yielding an initial distribution that is spatially homogeneous and uncorrelated. Previous studies confirm the existence of a continuous phase transition from an absorbing to an active phase at a particle density $\zeta_{c}=0.94885$ in one dimension [10], and 0.71695 in 2- $d$ [9]. For $\zeta<\zeta_{c}$, the stationary value of the order parameter (the density of active sites) is zero. 
I begin with the simplest case, relaxation of the order parameter $\rho$ in one dimension, for $\zeta<\zeta_{c}$. For $\zeta=0.5$, far below the critical value, the pattern of relaxation is essentially the same for systems of 1000 or more sites. Figure 1 shows that $\rho(t)$ (obtained from averages over $5 \times 10^{5}$ realizations of a system of 5000 sites), is well described by a stretched exponential, $\rho(t) \sim \exp \left[-\left(t / t_{0}\right)^{\beta}\right]$ with $\beta=0.45$ ( $t_{0}$ represents a characteristic timescale for relaxation). In this and subsequent analyses, the exponent $\beta$ is determined using the criterion of zero curvature, in the asymptotic region of the graph of $\ln [\rho(t)-\bar{\rho}]$ versus $t^{\beta}$ $\left(\bar{\rho}\right.$ is the asymptotic activity density, which is of course zero for $\zeta<\zeta_{c}$ ). A rough estimate of the uncertainty in $\beta$ values is \pm 0.02 .

Next I examine the relaxation for $\zeta=0.9$, much nearer the critical point. There are now considerable finite-size effects and much larger systems (up to $10^{5}$ sites) are required to observe the asymptotic behavior. For small systems $\rho(t)$ appears to decay faster than a stretched exponential, but as $L$ is increased, the long-time slow relaxation develops. For $L=10^{5}, \rho(t)$ again follows a stretched exponential, with $\beta=0.39$. It should be emphasized that while the simulation data cannot be interpreted as proving stretched-exponential relaxation, they do serve to rule out definitively both exponential and powerlaw relaxation of $\rho(t)$ in the subcritical regime.

In the supercritical regime, the relaxation of the order parameter to its stationary value $\bar{\rho}$ is nonmonotonic. $\rho(t)$ decays rapidly at first, and then slowly approaches the stationary value from below. For $\zeta=1$, I find $\bar{\rho}=0.118222(8)$, with $\Delta \rho \equiv \bar{\rho}-\rho(t)$ decaying asymptotically as a power-law, $\sim t^{-0.54}$ (see Fig. $2)$. The initial decay is again well described by a stretched exponential, with an exponent $\beta=0.28$. For somewhat higher densities $(\zeta=1.2$ and 1.3), the asymptotic approach to $\bar{\rho}$ (from below), is again via a power law, with an exponent of $0.5-0.52$.

I also studied a variant of the stochastic sandpile introduced in Ref. [19], in which the toppling rate at a site with $z$ particles is $z(z-1)$. (In this case $\zeta_{c}=0.9493(2)$.) For $p=1 / 2$, the decay to $\rho=0$ is described with high precision by a stretched exponential with $\beta=0.475(25)$. In the supercritical regime the approach to the stationary value is nonmonotonic, similar to that observed above. Unlike the constant-rate model, however, the relaxation is well described by an expression consisting of two stretched exponentials:

$$
\rho(t)=\bar{\rho}+A_{1} \exp \left[-\lambda_{1} t^{1 / 2}\right]-A_{2} \exp \left[-\lambda_{2} t^{1 / 2}\right],
$$

with $A_{1}, A_{2}, \lambda_{1}$, and $\lambda_{2}$ all positive constants $\left(A_{1} \gg A_{2}\right.$, and $\left.\lambda_{1} \gg \lambda_{2}\right)$.

Slow relaxation appears to be robust under changes in the toppling rate. Of equal interest is the nature of relaxation in two or more dimensions. At a density of $\zeta=0.5$ (well below $\zeta_{c}=0.71695(5)$ ), studies using lattices of $L^{2}$ sites, with $L=40,80$, and 160 , reveal that the order parameter decays as a stretched exponential, but with an exponent $\beta=0.81$. Nearer the critical point $(\zeta=0.7)$, finite-size effects are prominent, as was found in the onedimensional case. Studies using system sizes $L$ of up to 1280 again confirm stretched-exponential decay with $\beta=0.81$, as shown in Fig. 3 . 
The relaxation above the critical density (in a system of size $L=160$ at $\zeta=$ $0.75 ; \bar{\rho}=0.04995)$, is nonmonotonic, as in one dimension. The excess density is again well described by the difference between two stretched exponentials, as in Eq. (1), but in this case the exponent $\beta$ associated with the early decay is about 0.4 , while the long-time approach to $\bar{\rho}$ (from below) is characterized by an exponent of 0.8 (see Fig. 3, inset).

As we have seen, relaxation of the order parameter in the stochastic sandpile is characterized by stretched-exponential (or in some cases, powerlaw) functions. Recently, O'Donoghue and Bray [26] demonstrated stretchedexponential decay of the persistence probability, i.e., that a given site has never been visited by a diffusing particle, in certain one-dimensional reaction/diffusion processes. This suggests a study of persistence in the sandpile. In fact, since a site that is initially below threshold for toppling must be visited by another diffusing particle or particles before it can topple, persistence seems particularly relevant to sandpile relaxation dynamics. (Studies of dynamic critical exponents for persistence in conserved lattices gases, which are closely related to sandpiles, were reported by Lübeck [27]; here, however, we focus on the dynamics away from the critical point.)

In studying persistence in a sandpile, it appears useful to group sites according to their initial occupation number $z$. Let $p(t ; z)$ denote the persistence probability of sites whose initial height is $z$. For $z=0$ or 1 , I define $p(t ; z)$ as the probability that a site has not toppled up to time $t$. For $z>2$, however, such a definition is not very interesting, since (in the constant-rate model), the probability not to have toppled up to time $t$ is simply $e^{-t}$. For $z \geq 2$, therefore, $p(t ; z)$ is defined as the probability not to have toppled a second time. I study the one-dimensional fixed-rate model with particle densities $\zeta=0.5,0.9$, and 1 , as above.

For $\zeta<\zeta_{c}$, all activity ceases after a finite time, so that the persistence probability approaches a nonzero value $\bar{p}(z)$ at long times. For $\zeta=0.5$, the asymptotic relaxation of $p(t ; z)$ to $\bar{p}(z)$ again follows a stretched exponential; the best estimates for the exponent $\beta$ are 0.42 for $z=0$ and $\beta=0.39$ for $z=1$, 2 and 3. For $\zeta=0.9$, a study using $L=10^{4}$ yielded $\beta \simeq 0.44,0.47,0.49$, and 0.41 for $z=0,1,2$, and 3, respectively. Finally, for $\zeta=1$, the asymptotic decay of the persistence probability (studied on a ring of $2 \times 10^{4}$ sites) follows a stretched exponential with $\beta$ values of $0.42,0.41,0.38$, and 0.45 for the various $z$ values. The persistence probabilitites for $\zeta=0.9$ and $\zeta=1$ are shown in Fig. 4. (In all of these studies, the exponents for $z=2$ and 3 are less certain, due to poorer statistics, and to the fact that stretched-exponential behavior sets in later than for $z=0$ or 1 . I find no clear trend in the estimated exponent value, either with $z$ or with $\zeta$.) The foregoing results can be summarized as indicating that in one dimension, the asympotic relaxation of the persistence probability $p(t ; z)$ follows a stretched exponential with an exponent $\beta$ of about 0.42 .

The simulation results indicate that the relaxation of the order parameter and of the persistence probability, in a stochastic sandpile away from its crit- 
ical point, generically exhibits stretched-exponential scaling. The exponent $\beta$ generally takes values in the neighborhood of 0.4 in the one-dimensional case, while in two dimensions (for which only order-parameter relaxation was studied) the value is about 0.8 . Stretched-exponential relaxation may be understood as a consequence of the depletion of active elements, leading to a decreasing relaxation rate, $(1 / \rho)|d \rho / d t|$, as time goes on. The larger exponent in two dimensions is plausible, given the larger number of paths in configuration space. Detailed explanations of the streteched-exponential form, and of specific exponent values, remain as open challenges.

The observation of stretched-exponential relaxation suggests a connection with glassy dynamics. Superficially, the sandpile model seems to have little connection with dynamics of a dense fluid, but the two problems are related at a more abstract level. In dense fluids, not all the empty space in the system is available for particle movement, leading to a highly cooperative dynamics 28,29]. Some larger grouping of "voids" is required for relaxation on scales beyond that of the local cage. (For simplicity, I frame the analogy in terms of a hard-sphere fluid, in which the relevant variable is density not temperature.) Similarly, in a sandpile not all particles are available for movement (and relaxation): only those with companions in the same cell are mobile. These observations suggest a parallel between particles in the sandpile and parcels of unoccupied space in a dense fluid. The dynamic arrest in the fluid, when the density approaches an (apparent) transition value, corresponds to the absorbing-state phase transition in the sandpile. The associated order parameter - mobile free volume in the fluid - vanishes in the absorbing phase. (The total unoccupied volume is of course conserved, but the fraction that is mobile or "active" is not.) From this vantage, the liquid-glass transition corresponds to a dynamic transition, akin to directed percolation, of free volume, as opposed to the static percolation transition suggested some time ago by Cohen and Grest 30]. While the sandpile models studied to date seem too simplistic to capture the dynamics of a dense fluid near the glassy state, the results of the present study suggest that it would be worthwhile pursuing this analogy by developing somewhat more realistic particle models, and studying diffusion and the response to external driving, in the hope of gaining further insight into real glasses.

\section{Acknowledgments}

I thank Kim Christensen and Francisco F. Araujo Jr. for helpful discussions. This work was supported by CNPq, CAPES, and FAPEMIG, Brazil. 


\section{REFERENCES}

[1] BAK P., TANG C. and WIESENFELD K., Phys. Rev. Lett. 59 (1987) 381; Phys. Rev. A 38 (1988) 364.

[2] GRINSTEIN G., in Scale Invariance, Interfaces and Nonequilibrium Dynamics, (NATO Advanced Study Institute, Series B: Physics, vol. 344) edited by A. McKANE et al., (Plenum Press, New York) 1995.

[3] DHAR D., Physica A 263 (1999) 4, and references therein.

[4] DICKMAN R., MUÑOZ M. A., VESPIGNANI A., and ZAPPERI S., Braz. J. Phys. 30 (2000) 27; cond-mat/9910454 Preprint, 1999.

[5] MUÑOZ M. A., et al. in Procedings of the 6th Granada seminar on computational physics, edited by J. MARRO and P. L. GARRIDO, (American Institute of Physics, New York) 2001; cond-mat/0011447 Preprint, 2000.

[6] TANG C. and BAK P., Phys. Rev. Lett. 60 (1988) 2347.

[7] VESPIGNANI A. and ZAPPERI S., Phys. Rev. Lett. 78 (1997) 4793; Phys. Rev. E 57 (1998) 6345.

[8] MONTAKHAB A. and CARLSON J. M., Phys. Rev. E 58 (1998) 5608.

[9] VESPIGNANI A., et al., Phys. Rev. E 62 (2000) 4564.

[10] DICKMAN R., et al., Phys Rev. E64 (2001) 056104.

[11] LÜBECK S., Phys. Rev. E64 (2001) 016123; Eur. Phys. J. B26 (2002) 75.

[12] PRIEZZHEV V. B., J. Stat. Phys. 74 (1994) 955; IVASHKEVICH E. V., J. Phys. A 27 (1994) 3643; IVASHKEVICH E. V., KTITAREV D. V. and PRIEZZHEV V. B., Physica A 209 (1994) 347.

[13] ROSSI M., PASTOR-SATORRAS R. and VESPIGNANI A., Phys. Rev. Lett. 85 (2000) 1803.

[14] JANSSEN H. K., Z. Phys. 42 (1981) 141; ibid. 58 (1985) 311.

[15] GRASSBERGER P., Z. Physik. B 47 (1982) 465.

[16] PRIEZZHEV V. B., et al., Phys. Rev. Lett. 87 (2001) 084301.

[17] DICKMAN R., TOMÉ T. and DE OLIVEIRA M. J., Phys. Rev. E66 (2002) 016111.

[18] DICKMAN R., Phys. Rev. E, in press; cond-mat/0204608 Preprint, 2002.

[19] DICKMAN R. and VIDIGAL R. J. Phys. A35 (2002) 7269.

[20] MARRO J. and DICKMAN R., Nonequilibrium Phase Transitions in Lattice Models, (Cambridge University Press, Cambridge) 1999.

[21] LIGGETT T. M., Interacting Particle Systems, (Springer-Verlag, New York) 1985.

[22] JEAGER H. M., LIU C. and NAGEL S. R., Phys. Rev. Lett. 27 (1989) 40.

[23] FRETTE V., et al., Nature 397 (1996) 49.

[24] HEAD D. A. and RODGERS G. J., J. Phys. A 32 (1999) 1387.

[25] MANNA S. S., J. Stat. Phys. 59 (1990) 509; J. Phys. A 24 (1991) L363.

[26] O'DONOGHUE S. J. and BRAY A. J., Phys. Rev. E64 (2001) 041105.

[27] LÜBECK S. and MISRA A. Eur. Phys. J. B26 (2002) 75.

[28] POOLE P. H., DONATI C. and GLOTZER S. C., Physica A261 (1998) 51.

[29] DOLIWA B. and HEUER A., Phys. Rev. E61 (2000) 6898.

[30] COHEN M. H. and GREST G. S., Phys. Rev. B20 (1979) 1077. 


\section{FIGURE CAPTIONS}

FIG. 1. Relaxation of the order parameter in the one-dimensional sandpile, $\zeta=0.5, L=5000$. Inset: semi-log plot of the same data.

FIG. 2. Relaxation of the order parameter in the one-dimensional sandpile, $\zeta=1, L=2 \times 10^{4}$. Inset: log-log plot of the excess $\Delta \rho=\rho(t)-\bar{\rho}$.

FIG. 3. Relaxation of the order parameter in the two-dimensional sandpile, $\zeta=0.7, L=1280$. Inset: $\ln |\Delta \rho|$ versus $\ln t$ for $\zeta=0.75$; points: simulation; solid curve: fit using two stretched exponentials as described in text.

FIG. 4. Persistence probabilities $p(t ; z)$ for $z=0,1,2$, and 3 (upper to lower) in the one-dimensional model with $\zeta=1$. Inset: a similar plot for $\zeta=0.9$. 


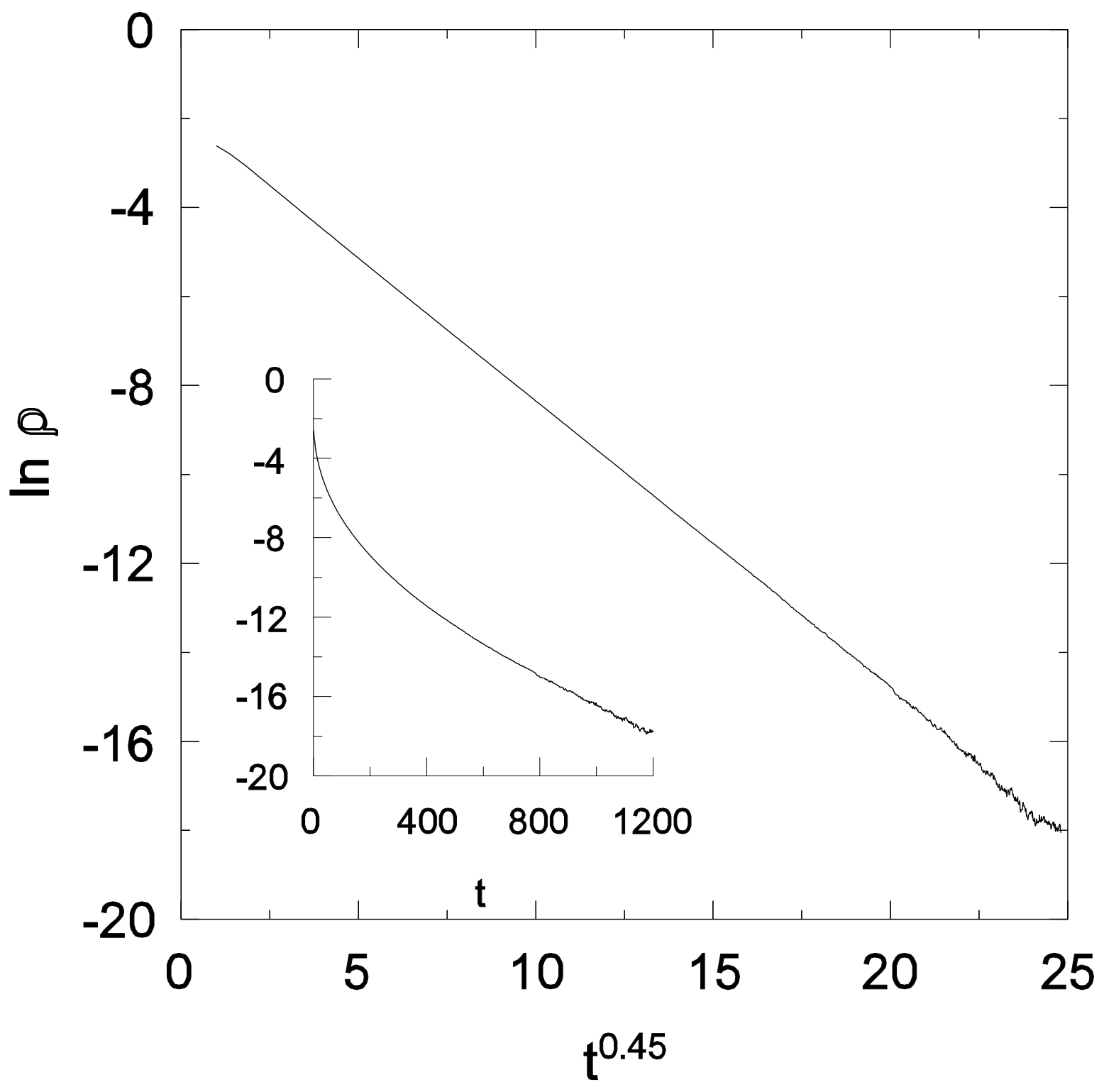




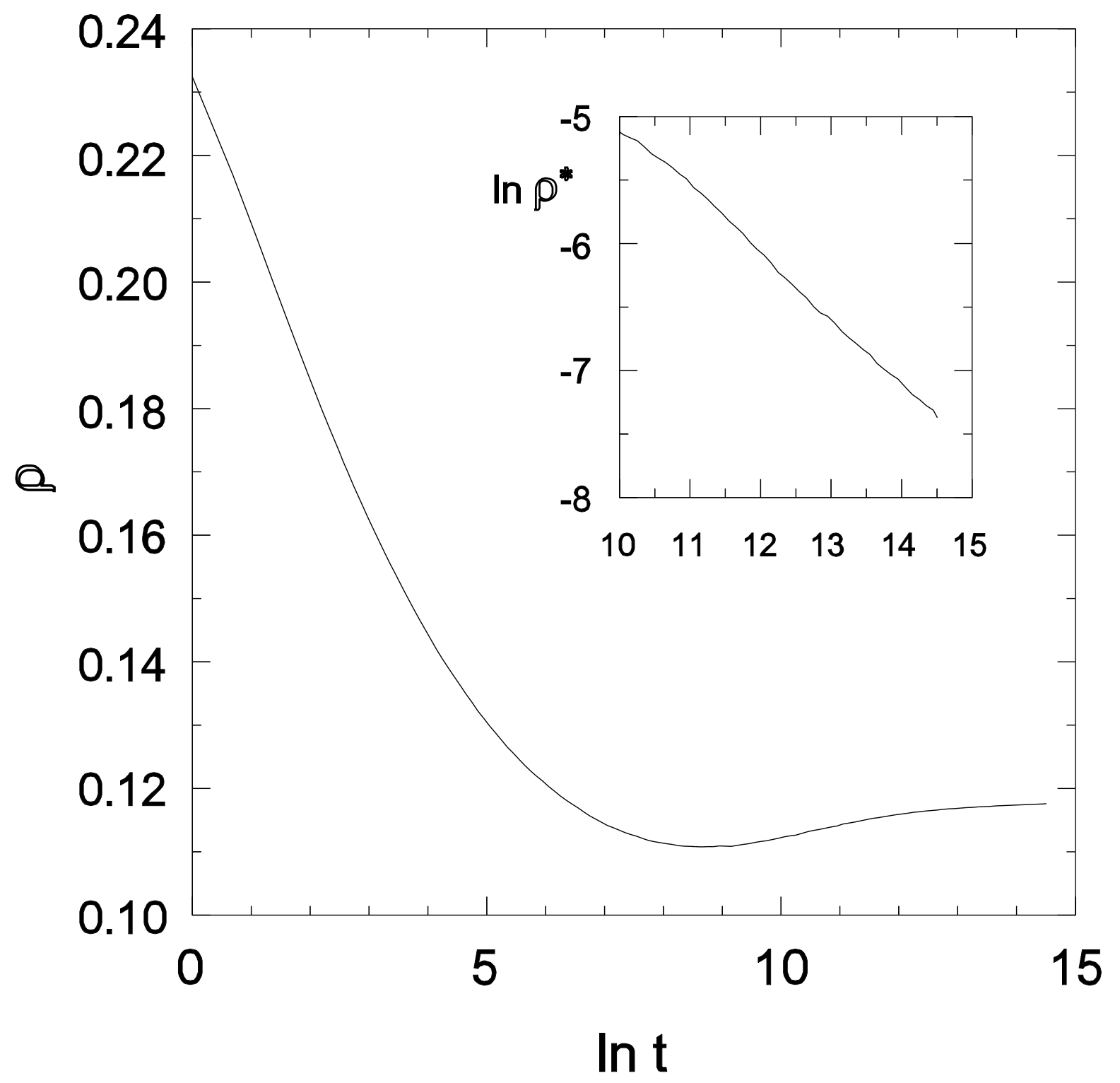




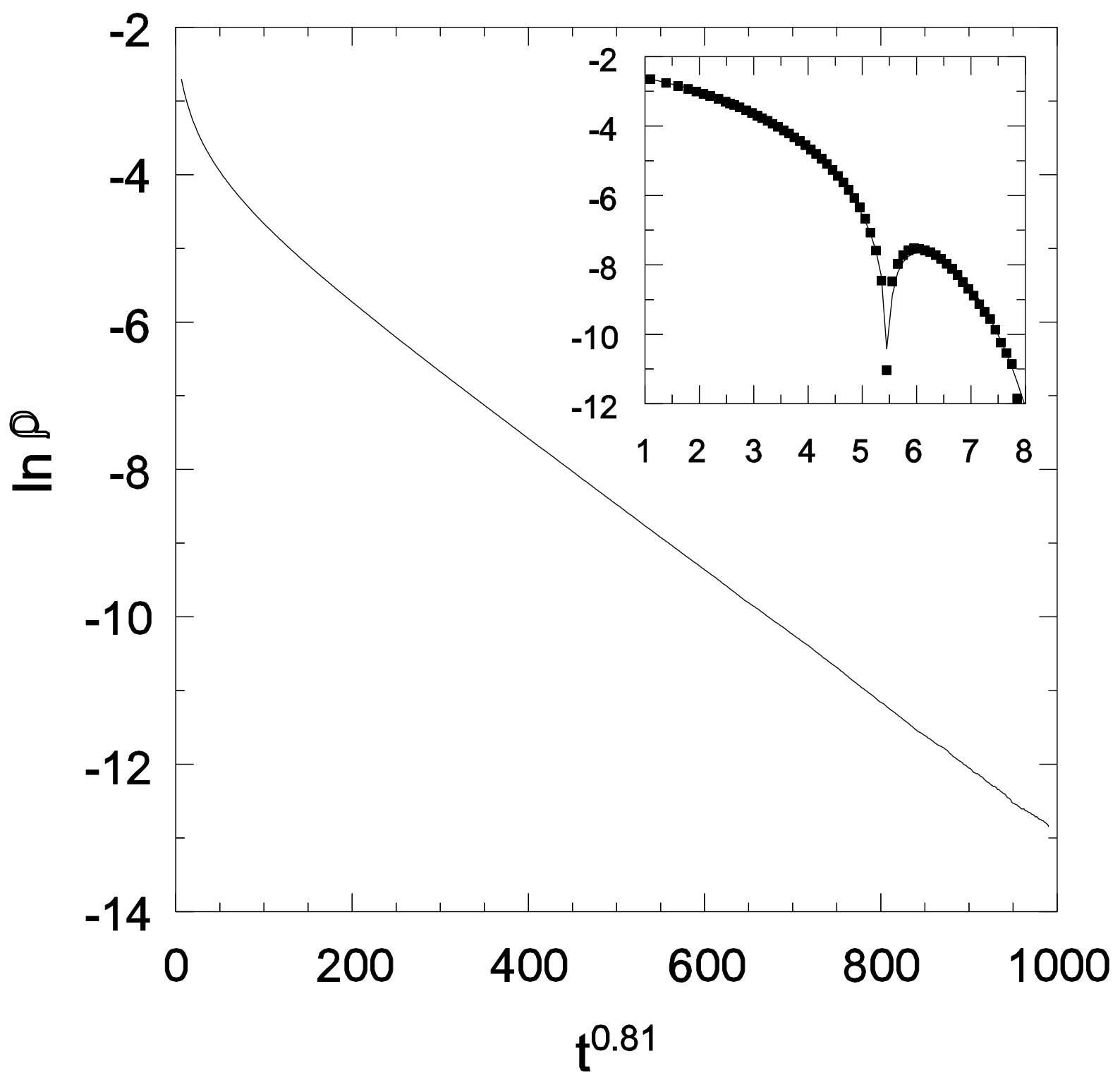




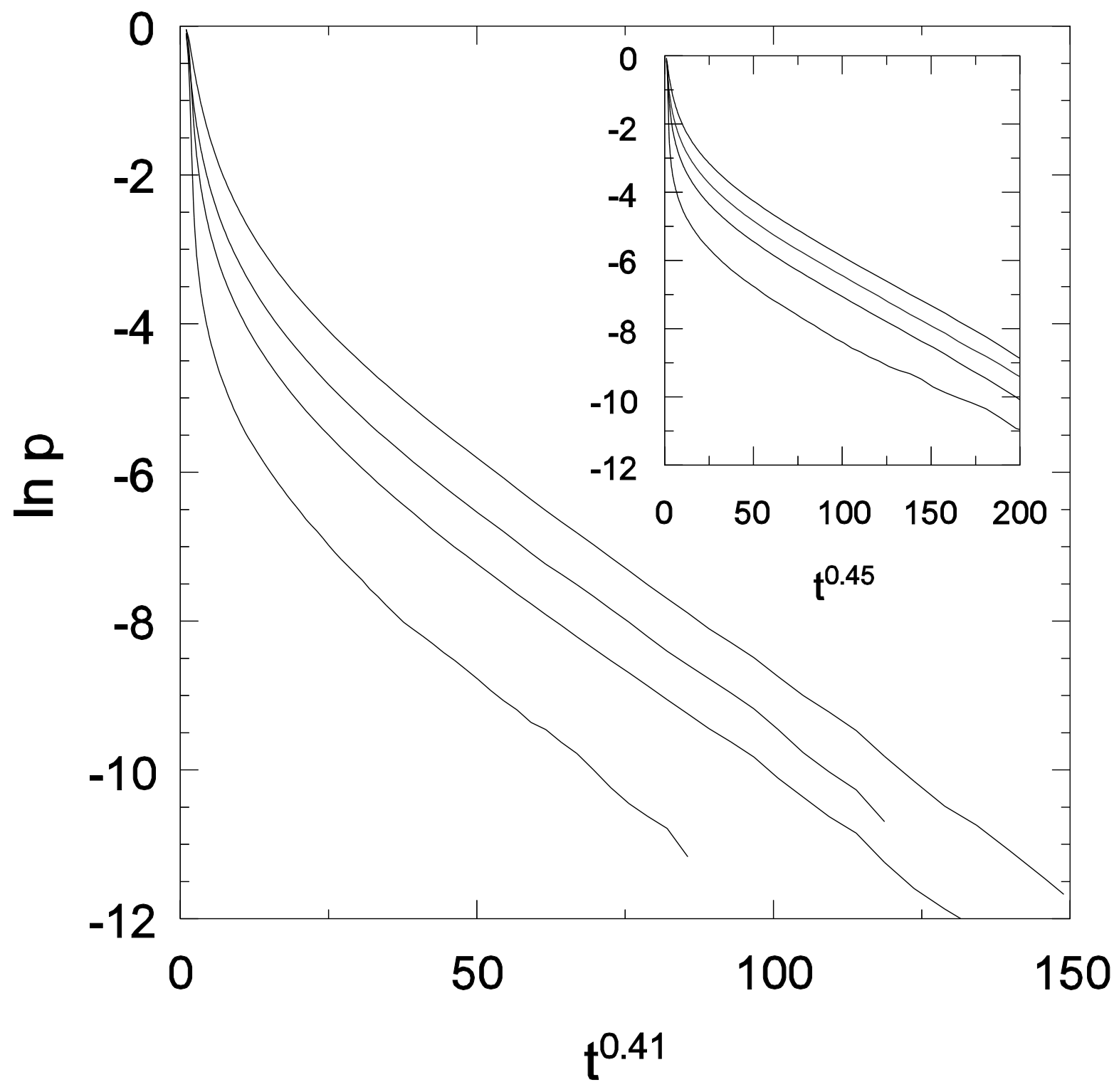

